\title{
Dual-task influences on retrieval from semantic memory and coordination dynamics
}

\author{
KEVIN SHOCKLEY \\ University of Cincinnati, Cincinnati, Ohio \\ and \\ M. T. TURVEY \\ University of Connecticut, Storrs, Connecticut
}

\begin{abstract}
Bimanual 1:1 rhythmic coordination was performed while retrieving words from a specified category. The effects of divided attention (DA) on coordination were indexed by changes in mean relative phase and recurrence measures of shared activity between the two limbs. Effects of DA on memory were indexed by deficits in exemplars retrieved relative to the baseline. Shifts in relative phase were found, accompanied by a recall deficit for DA during the retrieval task. DA also reduced the degree of shared activity between left and right rhythmic motions. Our discussion focuses on DA-induced parameter changes in retrieval and coordination dynamics, as well as on the hypothesis that stability is the general factor mediating dual-task performance.
\end{abstract}

Results from experiments in which a participant retrieves a previously studied word list while performing a task that does not involve memorization (e.g., a simple reaction time task or card sorting) have led to the suggestion that retrieval is "obligatory" (Craik, Govoni, NavehBenjamin, \& Anderson, 1996). The frequent observation from such experiments is that retrieval disrupts the parallel nonmemory task, but not vice versa; somewhat paradoxically, these results imply that retrieval is both (1) autonomous and (2) resource consuming (Anderson, 2001; Craik et al., 1996).

But although such observations are frequent, they are not ubiquitous. Retrieval has been found to suffer from parallel activity, with both the type of retrieval (see, e.g., Baddeley, Lewis, Eldridge, \& Thomson, 1984, Experiment 9) and the nature of the parallel activity (e.g., Fernandes \& Moscovitch, 2000) implicated as the reason for the deviation from the norm. Understandably, the question has arisen of the kind of theoretical framework that could accommodate such "seemingly disparate findings" (Anderson, 2001, p. 214).

Recently, Shockley and Turvey (2005) examined the effects when retrieving was conducted in parallel with

This work formed the basis of a doctoral dissertation completed by K.S. at the University of Connecticut. The research was supported in part by NSF Grants SBR 00-04097 and SBR 04-23036 to M.T.T. and by Williams College Division III funding awarded to K.S. We thank Michael Riley for his technical support and conceptual contributions, and we are especially thankful to the late Gerri Pellecchia (1956-2005), our dear friend and colleague, to whom we dedicate this article. Correspondence concerning this article should be addressed to $\mathrm{K}$. Shockley, Department of Psychology, Mail Location 376, University of Cincinnati, Cincinnati, OH 45221-0376 (e-mail: kevin.shockley@uc.edu). bimanual rhythmic activity. They did so because 1:1 frequency locking of two limbs offers a potentially rich set of dynamical measures for characterizing the performance changes induced by concurrent memory tasks. The nature of these measures could shed light on the class of operations underlying retrieving and/or the kind of system assembled to satisfy dual-task performance. Of particular significance are dynamical measures that characterize the attractor (i.e., the stable state) of the rhythmic coordination-namely, the attractor's location, strength, and noise (or, more precisely, the system noise associated with the particular stable state). Research has suggested that concurrent performance of a cognitive activity (e.g., counting backward by threes) shifts the stable state-the attractor-to a new value (i.e., one limb consistently leads the other by some new magnitude) and amplifies noise (i.e., the stable state fluctuates more), but leaves unaltered the attractor strength (i.e., how quickly the system settles back on the stable state following a perturbation) (Pellecchia, Shockley, \& Turvey, 2005). In extending the latter research to memory activity, Shockley and Turvey replicated the frequent observation that retrieval affects the parallel task, but not vice versa (and the frequent observation that encoding and the parallel task affect each other). Concurrent recall of a list of unrelated words shifted the coordination attractor and rendered it noisier without changing its stability; concurrent coordination had no effect, however, on the number of words recalled.

As noted, the frequent observation that retrieval is impervious to divided attention (DA) is not upheld by all retrieval tasks. Semantic retrieval, for example, has been shown to produce results congruent with DA at encoding: Both semantic retrieval and performance of the nonmemory task are impaired (Baddeley et al., 1984; 
Park, Smith, Dudley, \& Lafronza, 1989). In the present research, we investigated the retrieval task of generating category exemplars. As in Shockley and Turvey (2005), we used rhythmic coordination of left and right handheld pendulums as the nonmemory task. The coordination was in phase (the two pendulums moved forward and backward together with a relative phase of $0^{\circ}$ ) and conducted at a tempo dictated by a metronome. The coordination was either (1) performed at a high tempo with left and right pendulums that differed in length and, therefore, in their intrinsic frequencies, or (2) performed at a low tempo with left and right pendulums of the same length and, therefore, the same intrinsic frequency. The formalisms of bimanual coordination dynamics (see, e.g., Kelso, 1995; Pellecchia et al., 2005) relate the coordination's tempo to the strength of left-right coupling or cooperation (e.g., a slower tempo elicits greater coupling, and is thus less resource demanding), and the left-right difference in intrinsic frequencies relates to competition (a larger difference elicits greater competition, and is thus more resource demanding). In brief, the contrast between Conditions 1 and 2 above is between less stable coordination (LSC) and more stable coordination (MSC), respectively. On the basis of the various formalisms (e.g., Amazeen, Amazeen, \& Turvey, 1998; Haken, Kelso, \& Bunz, 1985; Kelso, 1995) and data (Pellecchia et al., 2005; Shockley \& Turvey, 2005), we expected that LSC's attractor (indexed by relative phase) should be farther from $0^{\circ}$, noisier, and weaker than MSC's attractor.

\section{METHOD}

\section{Participants}

Ten undergraduates at Williams College provided informed consent and were paid $\$ 10$ for participation. All participants were righthanded with normal or corrected-to-normal vision.

\section{Materials and Apparatus}

The handheld pendulums were aluminum rods with wood handles $(64,48$, and $34 \mathrm{~cm}$ in actual length) with gravitational eigenfrequencies $(f)$ of $0.8,1.0$, and $1.3 \mathrm{~Hz}$, respectively (where

$$
f=\frac{1}{2 \pi} \sqrt{\frac{l_{e}}{g}},
$$

$g$ is the gravitational constant, and $l_{e}$ is the simple pendulum length). The pendulums were combined according to $\Delta f=\left(f_{\text {left }}-f_{\text {right }}\right)$, yielding two $\Delta f$ (or left-right competition) values of 0 and $0.5 \mathrm{~Hz}$. Two $48-\mathrm{cm}$ pendulums were used for the $\Delta f=0 \mathrm{~Hz}$ condition. A metronome supplied the two coordination tempos of 0.5 and $1.5 \mathrm{~Hz}$. The combination of the higher tempo $(1.5 \mathrm{~Hz})$ with the higher competition $(\Delta f=0.5 \mathrm{~Hz})$ defined the LSC, and the combination of the lower tempo $(0.5 \mathrm{~Hz})$ with the lower competition $(\Delta f=0 \mathrm{~Hz})$ defined the MSC.

A 6-D Fastrak (Skill Technologies, Phoenix, Arizona) motion analysis system was used to record movement trajectories of the pendulums. System hardware included an electromagnetic transmitter and electromagnetic sensors. One sensor (60-Hz sampling rate) was attached to the top of each pendulum. A digital auditory metronome was used to mark the pace of the movement oscillation.

The following categories were used for the retrieval task: female first name, country, occupation or profession, article of clothing, part of a building, military title, animal with four feet, kind of money, and musical instrument (Battig \& Montague, 1969).

\section{Design}

There were 3 trials for each of five conditions, yielding a total of 15 trials divided in five blocks. The trials were randomized by block, with different blocks involving retrieval matched for normative mean number of items generated (based on the category norms reported in Battig \& Montague, 1969). The participants' verbal responses during category generation were recorded on audiotape. The practice period and data collection lasted a total of about $20 \mathrm{~min}$.

The trials involving coordination were conducted at two levels of stability. Relative to the MSC of zero competition $(\Delta f=0 \mathrm{~Hz})$ and slow tempo/stronger coupling (metronome pace of $0.5 \mathrm{~Hz}$ ), the LSC involving competition $(\Delta f=0.5 \mathrm{~Hz})$ and high tempo/weaker coupling (metronome pace of $1.5 \mathrm{~Hz}$ ) induces a positive shift from the required relative phase of $0^{\circ}$ (i.e., the left limb leads the right limb) and magnifies the standard deviation of relative phase (see Amazeen et al., 1998, for a summary).

The five attention conditions were full attention to MSC (FAMSC), full attention to LSC (FA-LSC), full attention to retrieval (FA-R), divided attention between MSC and retrieval (DA-MSC), and divided attention between LSC and retrieval (DA-LSC). The data from FA-MSC, FA-LSC, and FA-R trials served as baselines for comparisons with DA trials. FA-MSC and FA-LSC trials involved only bimanual rhythmic coordination, with no memory task (first row, Figure 1), and served as a control for coordination performance. FA-R trials involved participants' generating items from designated categories, with no coordination task (second row, Figure 1). They were advised of the category at trial initiation and were provided $30 \mathrm{sec}$ to generate as many items from the given category as possible. DA trials involved a combination of the coordination task and the retrieval task (third row, Figure 1). In DA trials, participants were asked to perform the coordination task, with either MSC or LSC, while simultaneously generating as many items from the given category as possible in $30 \mathrm{sec}$.

This overall design permitted a single-factor ANOVA to evaluate the role of attention on retrieval performance, in which attention consisted of the FA-R, DA-MSC, and DA-LSC conditions. For evaluation of coordination performance, the design submitted to a two-factor ANOVA, involving the factors attention (FA or DA) and stability (MSC or LSC).

\section{Procedure}

For all trials, participants were seated in a straight-backed wooden chair with their forearms, positioned midway between pronation and supination, resting on the armrests of the chair. Each hand extended beyond the armrest to permit free movement of the wrist joint. Each hand gripped the handle of a pendulum firmly, with the top of the handle flush with the top of the fist. In FA and DA trials involving coordination, the required movement of each handheld pendulum was a smooth, continuous oscillation in a plane parallel to the sagittal plane through radial and ulnar deviations of the wrist. The required coordination was in phase - both muscularly (left and right homologous muscles contracting simultaneously) and spatially (left and right hands moving in parallel planes in the same direction simultaneously). The sequences of events and their durations are depicted in Figure 1.

In all trials involving retrieval, the participants were instructed on the category and provided $30 \mathrm{sec}$ to generate as many items from that category as possible. In DA trials, a smooth comfortable rhythm was established prior to instruction on the category. Items were only counted if they fell within the designated category. The mean across trials for a participant was used in the ANOVA.

Prior to the experiment proper, the participants practiced the retrieval and bimanual coordination tasks separately and together, under all five conditions for a minimum of $15 \mathrm{sec}$ each. The participants were instructed that for DA trials, they should give neither task priority. The experiment consisted of 3 trials in each of the five experimental conditions, for a total of 15 trials. The Institutional Review Board of Williams College approved the procedure. 


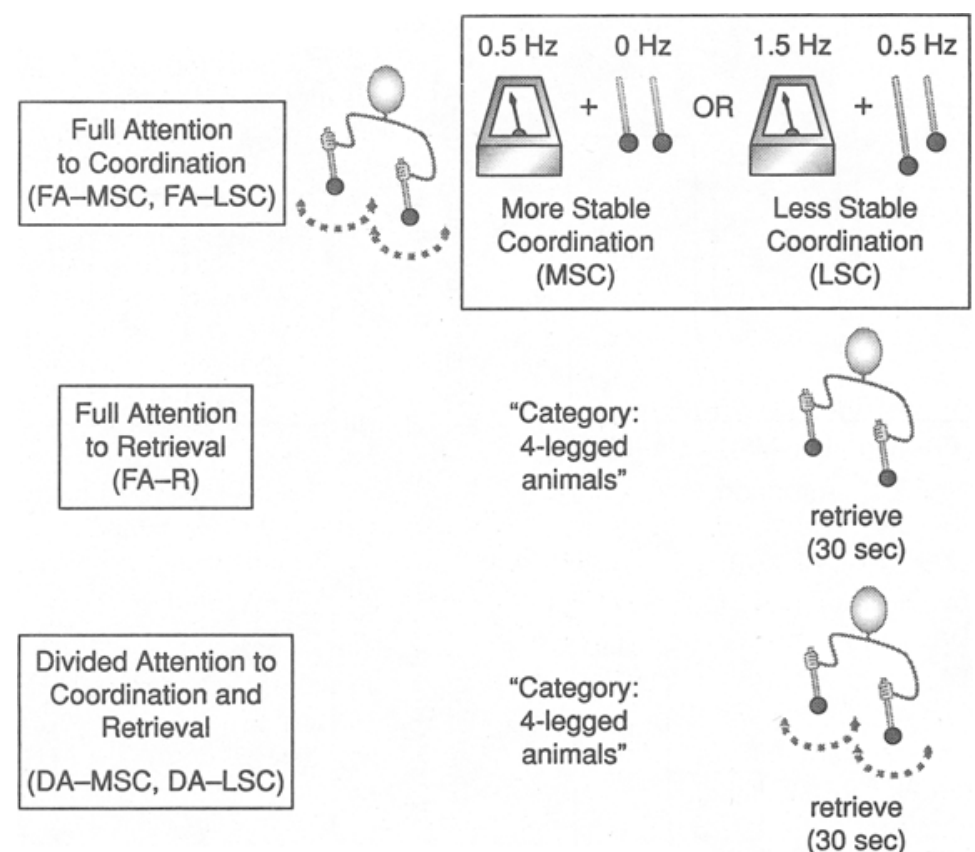

Figure 1. Design of the experiment (see text for details).

\section{Data Reduction}

Relative phase. Time series measuring horizontal displacement in the sagittal plane were collected from sensors positioned at the top of each pendulum. These were used to calculate phase angles as $\theta_{i}=\arctan \left(\dot{x}_{i} / \Delta x_{i}\right)$, where $\dot{x}_{i}$ is the angular velocity at sample $i$ (normalized in terms of the trial mean angular frequency) and $\Delta x_{i}$ is the angular displacement of sample $i$ (position at sample $i$ minus the trial mean position). For each trial, relative phase time series were computed as the difference between the left and right phase angles (i.e., $\left.\phi=\theta_{\text {left }}-\theta_{\text {right }}\right)$. The difference in phase angles averaged across a time series yielded $\langle\phi\rangle$ as the estimate of the attractor location-the magnitude by which one limb leads the other. For LSC in-phase coordination, $\langle\phi\rangle$ corresponds to a directionally specific shift of the attractor away from the required phase relation $\left(0^{\circ}\right)$. The variability in the phase angle difference across a time series yielded $S D \phi$ as an estimate of $\langle\phi\rangle$ 's variability and, thus, of the attractor location's variability.

Cross-recurrence quantification (CRQ). CRQ involves embedding a pair of time series in a multidimensional, time-delayed embedding space for the measurement of subtle time-correlated activity between the two signals (see, e.g., Richardson, Schmidt, \& Kay, in press; Shockley, Butwill, Zbilut, \& Webber, 2002; Webber $\&$ Zbilut, 1994). By tracking when values in one time series coincide with value(s) in the other time series (i.e., when the two time series are in similar states in embedding space), one can quantify the amount of shared activity-the percentage of cross-recurrence (\%REC). When the magnitude of noise (i.e., perturbations to the collective state) increases, the amount of shared activity (\%REC) decreases. The length of the longest sequence of recurrent states defines MAXLINE, and the inverse of MAXLINE provides an approximation of the largest positive Liapunov exponent manifest in a system's dynamics. These exponents have the general role of characterizing a system's stability/instability. By stability, we mean how quickly the collective state will return to the stable phase relation following a perturbation. Accordingly, a large MAXLINE can be interpreted as indicating less instability - that is, less divergence of the collective state in response to random perturbations - than a smaller MAXLINE (Zbilut, Zaldívar-Comenges, \& Strozzi, 2002). The lat- ter interpretation of MAXLINE and an interpretation of the inverse of \%REC as indexing system noise (and the relative independence of these measures) have been reinforced through simulations and experiments (e.g., Pellecchia et al., 2005; Richardson et al., in press). The parameters and procedures used for CRQ by Pellecchia et al. (2005) and Shockley and Turvey (2005) were used in the present experiment (i.e., embedding dimension $=5$; delay $=30$ and 10 data points [i.e., $1 / 4$ period] for 0.5 and $1.5 \mathrm{~Hz}$, respectively; radius = $20 \%$ maximum distance separating points in reconstructed space).

\section{RESULTS}

The results for the memory component are summarized in Figure 2A. Semantic retrieval was reduced by attention $\left[F(2,18)=9.20, p<.01, \eta^{2}=.51\right]$. The reduction, however, was not dependent on the stability of coordination, since the reduction due to LSC was not significantly greater than that due to MSC $(p>.05)$.

The results for coordination are summarized in Figures $2 \mathrm{~B}-2 \mathrm{~F}$. First, in both FA and DA conditions, the left and right oscillations did not differ $(F<1)$ and corresponded to the prescribed metronome frequencies $(0.5$ and $1.5 \mathrm{~Hz}$ ) (Figure 2B). Second, the effect of attention on $\langle\phi\rangle$ depended on stability $\left[F(1,9)=7.97, p<.05, \eta^{2}=\right.$ $.53]:\langle\phi\rangle$ in LSC was displaced farther from $0^{\circ}$ than $\langle\phi\rangle$ in MSC $(p<.05)$, with the magnitude of the difference greater for DA than for FA $(p<.05)$ (Figure 2C). Third, $S D \phi$ was greater for LSC than for MSC $[F(1,9)=5.46$, $p<.05, \eta^{2}=.73$ ], but $S D \phi$ was not greater for DA than for FA, and there was no attention $\times$ stability interaction $(F \mathrm{~s}<1)$ (Figure 2D). Fourth, MAXLINE was greater for MSC than for LSC $\left[F(1,9)=117.39, p<.01, \eta^{2}=.93\right]$ but not greater for DA than for FA, and the attention $X$ stability interaction was not reliable $(F \mathrm{~s}<1)$ (Figure 2E). 

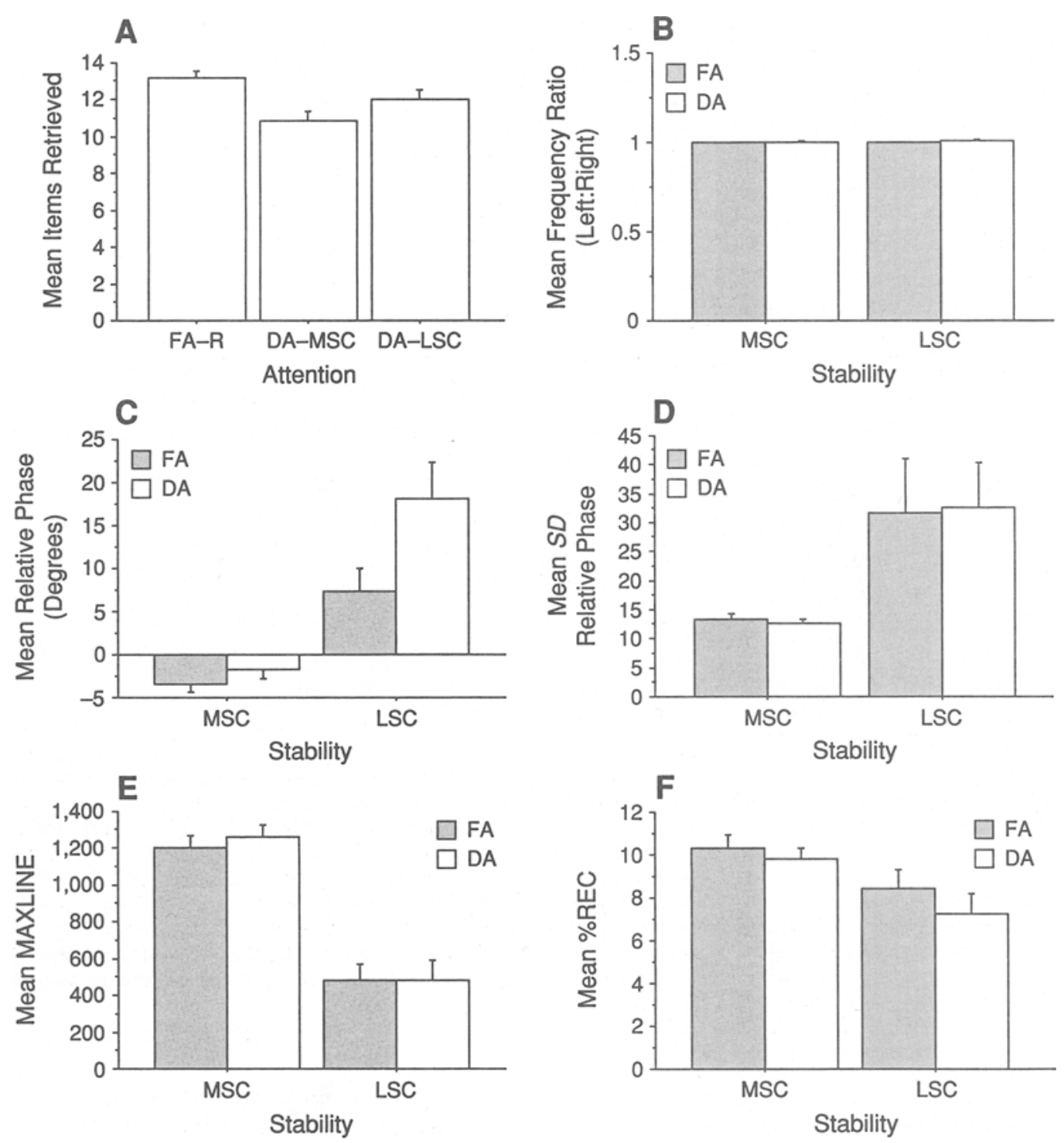

Figure 2. Effects of divided attention on retrieval and coordination. The panels show the influence of attention and stability on (A) mean items retrieved, (B) mean frequency ratio (left:right), (C) mean $\langle\phi\rangle$, (D) mean $S D \phi$, (E) mean MAXLINE, and (F) mean \%REC. See Figure 1 for the meanings of abbreviations.

Fifth, \%REC was greater for MSC than for $\operatorname{LSC}[F(1,9)=$ $\left.9.17, p<.05, \eta^{2}=.50\right]$ and for FA than for DA $[F(1,9)=$ $\left.9.69, p<.05, \eta^{2}=.52\right]$, but stability and attention did not interact $(F<1)$ (Figure $2 \mathrm{~F}$ ).

\section{DISCUSSION}

The results of the present experiment indicate that when retrieval from semantic memory and bimanual rhythmic coordination are conducted simultaneously for $30 \mathrm{sec}$, retrieval is impaired and the dynamics of coordination are altered.

The reduction of semantic retrieval by concurrent rhythmic coordination was not dependent on the stability of the coordination. LSC and MSC reduced retrieval to the same degree, consonant with the results for coordina- tion performed simultaneously with encoding (Shockley \& Turvey, 2005).

The reciprocal effect of semantic retrieval on coordination dynamics followed the pattern seen for encoding and episodic retrieval (Shockley \& Turvey, 2005) and for arithmetic activity (Pellecchia et al., 2005). Retrieval shifted the attractor location $(\langle\phi\rangle)$ of LSC but not of MSC, and it magnified the system noise (\%REC) in equal degree for LSC and MSC. Retrieval did not, however, alter the stability strength (MAXIINE values) of either LSC or MSC.

None of the identified sources of specific intertask interference were active in the present experiment (Bourke, Duncan, \& Nimmo-Smith, 1996). If a general rather than a specific factor mediated the present dual-task effects (Bourke, 1997; Bourke et al., 1996), one would have to 
assume that both the retrieval and coordination measures reflected that factor. As a candidate general factor, limited resources seems too coarse a hypothesis to address the intricate pattern of mutual influences between semantic retrieval and interlimb rhythmic coordination. A more promising candidate hypothesis for the general factor is stability. A temporally stable organization characterizes 1:1 frequency locking, and by hypothesis, it also characterizes semantic retrieval. The 30 -sec remembering task of the present experiment might entail an organized state encompassing (1) specification of the required category, (2) searching and finding exemplars, (3) evaluating retrieved exemplars for appropriateness, and (4) editing out repetitions (Baddeley, 1998; Koriat, 2000). Collectively, the foregoing monitoring and control processes constitute a retrieval mode that would have to be understood, like the coordination, in dynamical terms. Dual-task performance would then be the coupling of the two dynamics into a single stable organization.

Semantic retrieval has characteristic dynamics, as noted originally by Bousfield and Sedgewick (1944). It is most clearly evident at long time scales (e.g., retrieving for $20 \mathrm{~min}$ ), but it is also visible over shorter intervals of recall (e.g., $3 \mathrm{~min}$; Herrmann \& Murray, 1979). The rate of generating category exemplars begins rapidly and progressively decreases as the number of generated exemplars increases toward the participant's limit. This exponential retrieval can be derived from

$$
\dot{n}=(r / S)(N-n),
$$

where $\dot{n}$ is the rate of recall, $r$ is the rate at which items are sampled from the semantic set of size $S, N$ is the asymptotic number recalled, and $n$ is the number recalled at time $t$ (Wixted \& Rohrer, 1994).

The dynamics of bimanual rhythmic coordination with variable relative phase has the form ${ }^{1}$

$$
\begin{aligned}
\dot{\phi}= & \Delta \omega-a \sin \phi-2 b \sin 2 \phi \\
& -c \cos \phi-2 d \cos 2 \phi+\sqrt{Q} \cdot \xi_{t} .
\end{aligned}
$$

The parameters $a, b$ and $c, d$, respectively, determine the relative strengths of the stable attractive states at or near $\phi=0^{\circ}$ (in-phase coordination) and $\phi=180^{\circ}$ (antiphase coordination). Equation 2's final right-hand term is a Gaussian white noise process $\xi_{t}$ of strength $Q>0$. Theory and research suggest that $a, b$ and $\Delta \omega$ (in radians, equivalent to $\Delta f$ in cycles/sec) are primarily responsible for the coordination pattern (see, e.g., Amazeen et al., 1998; Haken et al., 1985; Kelso, 1995), with $c$ and $d$ playing more subtle symmetry-breaking roles than $\Delta \omega$ (Pellecchia et al., 2005; Treffner \& Turvey, 1995). Simulations show that changing $c$ with other parameters constant (as when retrieval is added to the coordination task) shifts attractor location with negligible change in attractor strength. An attractor location is identified by a zero crossing of negative slope in the plot of Equation 2 against $\phi$, and attractor strength is identified by the slope's magnitude $\lambda$. Formally, the variance $S D \phi$ associated with an attractor varies inversely with $\lambda$ and directly with $Q$ (Schöner, Haken, \&
Kelso, 1986). In the present research, $Q$ was measured by $1 / \%$ REC and $\lambda$ by MAXLINE. Although LSC relative to MSC increased $S D \phi$, increased 1/\%REC (i.e., increased $Q$ ), and decreased MAXIINE (i.e., decreased $\lambda$ ), ${ }^{2}$ semantic retrieval affected only $1 / \%$ REC and did so independently of the stability difference between LSC and MSC. Congruent with prior observations on encoding, episodic retrieval, and mental arithmetic, semantic retrieval added a fixed increment to $Q$. In sum, semantic retrieval, like the other cognitive activities studied to date, reparameterized the coordination dynamics of Equation 2 by changing $c$ and $Q$.

In simplest form, the retrieval dynamics can be expressed as $\dot{n}=R\left(n, \chi_{\text {ret }}\right.$; noise $)$ and the coordination dynamics as $\dot{\phi}=C\left(\phi, \chi_{\text {coor }} ;\right.$ noise $)$, with $\chi$ designating the control parameters. From the hypothesis that dual-task performance of semantic retrieval and coordination is interpretable as a coupling of the two dynamics, the coupling might be expected to entail parameter changes in retrieval dynamics paralleling the identified parameter changes in coordination dynamics. Could concurrent coordination dynamics lower the sampling rate $r$ or extend (defocus) the boundaries of the search set $S$ ? Either change (see Equation 1) in the present experiment would have affected the dependence of $\dot{n}$ on $n$ (Maylor, Chater, \& Brown, 2001; Wixted \& Rohrer, 1994) and reduced the number of exemplars generated in the 30-sec recall period. A first step toward evaluating the preceding ideas would be new experiments in which researchers (1) manipulated both retrieval dynamics (e.g., large and small semantic categories) and coordination dynamics (e.g., LSC and MSC); (2) examined several retrieval/coordination intervals (e.g., up to $3 \mathrm{~min}$; Herrmann \& Murray, 1979); (3) considered in addition to $\dot{n}$ the interresponse time (IRT) of exemplar generation, given the approximation $\operatorname{IRT}_{i}=S /[r(N-i)]$, where $i$ denotes position in the sequence of responses (Wixted \& Rohrer, 1994); and (4) performed a local, moving-window analysis to determine whether IRT covaries with the dynamical measures in question.

In sum, the primary observation of the present experiment, that cumulative retrieval and rhythmic coordination are mutually influential, opens the door on continuous dualtask assessments of memory phases in terms of stability measures. Such assessments can be expected to promote new methods of inquiry and new directions for theory development. In a wider perspective, such assessments can be expected to pose special challenges for modeling mutual influences between concurrent tasks (Pellecchia et al., 2005; Pellecchia \& Turvey, 2001; Temprado, 2004).

\section{REFERENCES}

Amazeen, P. G., Amazeen, E. L., \& Turvey, M. T. (1998). Dynamics of human intersegmental coordination: Theory and research. In D. A. Rosenbaum \& C. E. Collyer (Eds.), Timing of behavior: Neural, psychological, and computational perspectives (pp. 237-259). Cambridge, MA: MIT Press.

ANDERSON, N. D. (2001). The attentional demands and attentional control of encoding and retrieval. In M. Naveh-Benjamin, M. Moscovitch, \& H. L. Roediger III (Eds.), Perspectives on human memory 
and cognitive aging: Essays in honour of Fergus Craik (pp. 208-225). Philadelphia: Psychology Press.

BADDELEY, A. D. (1998). Human memory: Theory and practice. Boston: Allyn \& Bacon.

Baddeley, A. D., Lewis, V., Eldridge, M., \& Thomson, N. (1984). Attention and retrieval from long-term memory. Journal of Experimental Psychology: General, 113, 518-540.

BatTIG, W. F., \& MONTAGUE, W. E. (1969). Category norms for verbal items in 56 categories: A replication and extension of the Connecticut category norms. Journal of Experimental Psychology Monographs, 80(3, Pt. 2), $1-46$

BouRKE, P. A. (1997). Measuring attentional demand in continuous dual-task performance. Quarterly Journal of Experimental Psychology, 50A, 821-840.

BOURKE, P. A., DUNCAN, J., \& Nimmo-SMITH, I. (1996). A general factor involved in dual-task performance decrement. Quarterly Journal of Experimental Psychology, 49A, 525-545.

Bousfield, W. A., \& SEDGewick, C. H. W. (1944). An analysis of sequences of restricted associative responses. Journal of General Psychology, 30, 149-165.

Craik, F. I. M., Govoni, R., Naveh-Benjamin, M., \& Anderson, N. D. (1996). The effects of divided attention on encoding and retrieval processes in human memory. Journal of Experimental Psychology: General, 125, 159-180.

DAFFerTShofer, A., van deN Berg, C., \& BeEk, P. J. (1999). A dynamical model for mirror movements. Physica $D, 132,243-266$.

FERNANDES, M. A., \& Moscovitch, M. (2000). Divided attention and memory: Evidence of substantial interference effects at retrieval and encoding. Journal of Experimental Psychology: General, 129, 155176.

HAKEN, H., Kelso, J. A. S., \& BUNZ, H. (1985). A theoretical model of phase transitions in human hand movements. Biological Cybernetics, $51,347.356$

HerrmanN, D. J., \& MurRay, D. J. (1979). The role of category size in continuous recall from semantic memory. Journal of General Psychology, 101, 205-218.

Kelso, J. A. S. (1995). Dynamic patterns. Cambridge, MA: MIT Press.

KorIAT, A. (2000). Control processes in remembering. In E. Tulving \& F. I. M. Craik (Eds.), The Oxford handbook of memory (pp. 333-346). New York: Oxford University Press.

MAYlor, E. A., Chater, N., \& Brown, G. D. A. (2001). Scale invariance in the retrieval of retrospective and prospective memories. Psychonomic Bulletin \& Review, 8, 162-167.

Park, D. C., SMith, A. D., Dudley, W. N., \& Lafronza, V. N. (1989). Effects of age and a divided attention task presented during encoding and retrieval on memory. Journal of Experimental Psychology: Learning, Memory, \& Cognition, 15, 1185-1191.

Pellecchia, G., Shockley, K., \& Turvey, M. T. (2005). Concurrent cognitive task modulates coordination dynamics. Cognitive Science, 29, 531-557.
Pellecchia, G., \& TURvey, M. T. (2001). Cognitive activity shifts the attractors of bimanual rhythmic coordination. Journal of Motor Behavior, 33, 9-15.

Richardson, M. J., Schmidt, R. C., \& KAY, B. A. (in press). Distinguishing the noise and attractor strength of coordinated limb movements using recurrence analysis. Biological Cybernetics.

SCHÖNER, G., HAKEN, H., \& Kelso, J. A. (1986). A stochastic theory of phase transitions in human hand movement. Biological Cybernetics, $53,247-257$

Shockley, K., Butwill, M., Zbilut, J. P., \& Webber, C. L., Jr. (2002). Cross recurrence quantification of coupled oscillators. Physics Letters $A, 305,59-69$.

SHOCKLEY, K., \& TURVEY, M. T. (2005). Encoding and retrieval during bimanual rhythmic coordination. Journal of Experimental Psychology: Learning, Memory, \& Cognition, 31, 980-990.

TEMPrado, J. J. (2004). A dynamical approach to the interplay of attention and bimanual coordination. In V. K. Jirsa \& J. A. S. Kelso (Eds.), Coordination dynamics: Issues and trends. Vol. 1: Applied complex systems (pp. 21-39). New York: Springer.

Treffner, P. J., \& TurveY, M. T. (1995). Handedness, and the asymmetric dynamics of bimanual rhythmic coordination. Journal of Experimental Psychology: Human Perception \& Performance, 21, 318-333.

WebBer, C. L., JR., \& ZBILUT, J. P. (1994). Dynamical assessment of physiological systems and states using recurrence plot strategies. Journal of Applied Physiology, 76, 965-973.

WIXTED, J. T., \& RoHRER, D. (1994). Analyzing the dynamics of free recall: An integrative review of the empirical literature. Psychonomic Bulletin \& Review, 1, 89-106.

Zbilut, J. P., Zaldivar-Comenges, J.-M., \& STrozzi, F. (2002). Recurrence quantification based Liapunov exponents for monitoring divergence in experimental data. Physics Letters A, 297, 173-181.

\section{NOTES}

1. Except for the cosine functions, Equation 2 is the Haken-KelsoBunz equation derived originally for $\Delta \omega(\mathrm{rad})=0[\Delta f(\mathrm{~Hz})=0]$ (Haken et al., 1985) and subsequently extended to $\Delta \omega \neq 0$. The cosine functions were motivated by hand asymmetry (Treffner \& Turvey, 1995) A formal motivation is presented in Daffertshofer, van den Berg, and Beek (1999).

2. The MAXLINE decline is expected because of the relations between $S D \phi$ and $\lambda$ (inverse) and $Q$ (direct) (Schōner et al., 1986). Values of $\lambda$ for Equation 2's attractors decline from the conditions defining MSC to the conditions defining LSC (see Pellecchia et al., 2005, Figure 1).

(Manuscript received August 9, 2005; revision accepted for publication April 11, 2006.) 